ISSN 2519-7398 (Versión electrónica)

DOI: http://dx.doi.org/10.21704/ac.v78i2.1056

(C) Universidad Nacional Agraria La Molina, Lima - Perú

\title{
Caracterización de la infiltración de agua en tres sistemas de uso del suelo de la Comunidad Santiago de Carampoma, Huarochirí, Lima
}

\author{
Characterization of infiltration of water in three systems of soil use of the peasant Community \\ Santiago de Carampoma, Huarochiri, Lima
}

\author{
Fernando Yáñez Gutiérrez*; Rosa María Hermoza Espezúa²; Luis Rubén Bazán Tapiaª \\ *Autor de correspondencia
}

\begin{abstract}
Resumen
Se caracteriza la infiltración en tres formas de uso del suelo: bosque natural intervenido, pastizal parcialmente compactado por sobrepastoreo y cultivo de papas en andenes agrícolas, dentro del área del bosque natural relicto de Japaní, perteneciente a la Comunidad Campesina Santiago de Carampoma, Huarochirí, Lima. Los datos se colectaron en el mes de agosto del año 2016 (época seca), utilizando el método del cilindro infiltrómetro individual (método del anillo simple). Se comparó el comportamiento de la infiltración en los tres usos de suelo mencionados, considerando además tres rangos de pendiente fisiográfica (alta, media, baja) y de rango altitudinal (alto, medio, bajo), adicionalmente se realizó el análisis de suelo. Los resultados mostraron que, los mayores valores de infiltración se dieron en las más altas pendientes fisiográficas, con los máximos en el bosque intervenido. La pendiente fisiográfica, especialmente sobre el $60 \%$, resultó ser el factor que más influyó en el incremento de la infiltración. La altitud solo mostró un efecto significativo al actuar junto con el factor pendiente fisiográfica.
\end{abstract}

Palabras clave: Cilindro infiltrómetro; Uso del suelo; Hidrología; Infiltración; Bosque relicto; Polylepis.

\begin{abstract}
This study characterizes the infiltration in three forms of land use: intervened forest, pasture partially compacted by overgrazing and potatoe crop in andean agricultural terraces, within the relict natural forest of Japaní, in the Peasant Community of Santiago de Carampoma, Huarochirí, Lima. The information was colleted in the month of August of the year 2016 (dry season). The individual infiltrator cylinder or simple ring method was used and the behavior of the infiltration in the forest, the pasture and the andean terraces was compared in three types of physiographic slope (high, medium, low); and of altitudinal range (high, medium and low, expressed in meters above sea level). Additionally, soil analysis was done. The results showed that the highest values of infiltration occurred in the highest physiographic slopes, with the maximum reached in the intervened forest. The physiographic slope, especially the one of $60 \%$, proved to be the most influential factor in infiltration. Altitude only showed a significant effect when acting along with the physiographic slope factor.
\end{abstract}

Keywords:Cylinder infiltrometer; Land use; Hydrology; Infiltration; Relict forest; Polylepis.

\section{Introducción}

Aquafondo (2016) enuncia que, tras la ciudad de El Cairo en Egipto, Lima, capital del Perú, con cerca de 10 millones de habitantes, es la segunda ciudad costera más grande del mundo desarrollada dentro de un desierto. Según el INEI (2017) Lima posee un promedio de precipitación anual aproximado de 7,7 milímetros, sin grandes ríos ni reservas de agua superficial, siendo la disponibilidad de agua per cápita inferior al límite crítico establecido por la $\mathrm{FAO}$ de $1700 \mathrm{~m}^{3} /$ habitante/año (FAO, 2017) . Vásquez (2011) estimó que para el 2050, el mayor incremento poblacional se dará en está región. Este crecimiento viene de la mano con la expansión económica, que repercute en el aspecto social y ambiental, generando motivos suficientes para deducir que muy probablemente con el tiempo se presenten graves conflictos sociales y políticos a causa de la acentuada escasez de agua y la disminución del acceso a este importante recurso.

El problema se agravaría debido a que dicho crecimiento urbano-económico, también está presente en las cuencas hidrográficas altoandinas, influenciando en la descarga de agua hacía zonas costeras, destacando desde hace cientos de años la ocurrencia de acciones antrópicas como el sobrepastoreo y la deforestación en las partes altas y medias

\footnotetext{
${ }^{1}$ Consultor independiente, Lima, Perú. Email: fernandu2007@hotmail.com; 20081333@lamolina.edu.pe

${ }^{2}$ Facultad de Ciencias Forestales, Universidad Nacional Agraria la Molina, Lima, Perú. Email:. rosamaria@lamolina.edu.pe

${ }^{3}$ Facultad de Agronomía, Universidad Nacional Agraria la Molina, Lima, Perú. Email:. rbt@lamolina.edu.pe
} 
de las cuencas, causando según Reyes (2014) las variaciones en los regímenes hidrológicos, perdida de la fertilidad, salinidad, erosión hídrica y arrastre masivo del suelo.

Sin embargo, parte de la paulatina disminución de dichos problemas se encuentra en la conservación, protección y restauración de los bosques altoandinos. Herzog et al, 2011; citado por Doornbos (2015) destaca el rol que desempeñan en la regulación hídrica, el rendimiento hídrico y la protección del suelo contra el impacto de la lluvia, reduciendo la erosión y los peligros de deslizamientos, y manteniendo así la calidad del agua. Adicionalmente, ECOAN, 2007; citado por Gonzalez (2015) destaca el papel principal de estos bosques como depósitos de agua, almacenando una gran cantidad proveniente de la lluvia y de las densas nieblas a las que están sometidos. Debido a todo ello y conociendo el papel del bosque natural relícto de Japaní, de las actividades económicas como la agricultura y la ganadería en la zona, y el impacto de estas actividades en la regulación del caudal y del balance hidrológico de la zona media y alta de la microcuenca del río Suncha, que a su vez se encuentra formando parte de la subcuenca del río Santa Eulalia, uno de los principales tributarios del río Rímac, (abastecedor de agua a Lima y Callao); el presente estudio tiene la finalidad de contribuir en caracterizar y comparar el grado de influencia relativa del bosque intervenido de Japaní en la infiltración del agua de las lluvias en el suelo, en contraste con otros sistemas de uso de la tierra, así como evaluar el proceso de infiltración en cada sistema de uso de suelo, mediante el método del cilindro infiltrómetro individual, durante el periodo seco.

\section{Área de estudio}

El bosque natural relicto de Japaní ocupa un área aproximada de 665 hectáreas (Sembrero \& Valencia, 2015), y se encuentra ubicado en la parte media de la microcuenca del río Suncha. Esta microcuenca en conjunto con la comunidad campesina Santiago de Carampoma pertenece al distrito de Carampoma, Provincia de Huarochiri, Departamento de Lima (Figura 1).

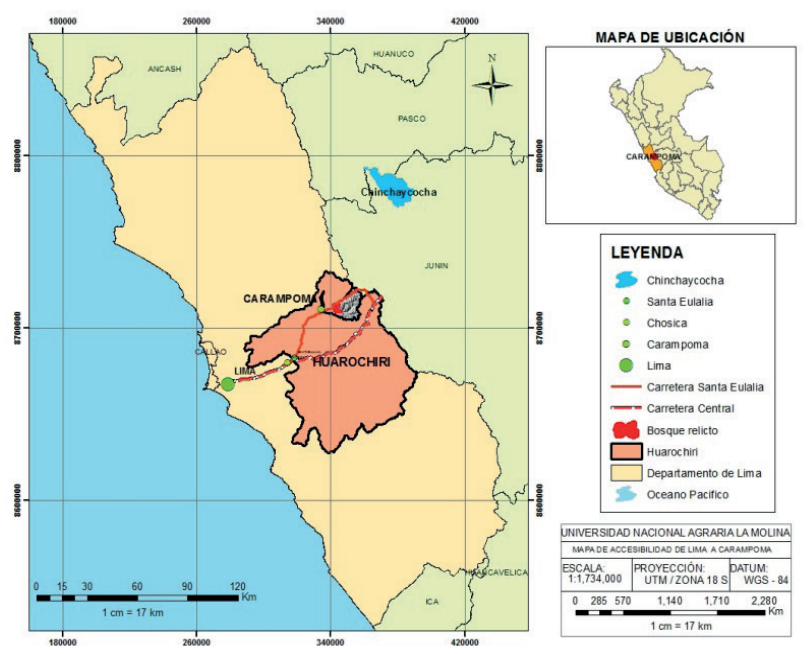

Figura 1. Ubicación del Bosque natural relicto de Japaní (Huarochiri, Lima)
La zona se ubica entre los 3600 y $4100 \mathrm{msnm}$, presenta un clima frio, con una temperatura media anual de $15,3^{\circ} \mathrm{C}$, siendo su máxima media anual $19,8^{\circ} \mathrm{C}$ y su mínima media anual $10,9^{\circ} \mathrm{C}$. La media de precipitación total anual varía de $500 \mathrm{~mm}$ a $700 \mathrm{~mm}$, reportándose valores promedio de precipitación mensual de $0,3 \mathrm{~mm}$ en época seca (abril a septiembre) y 95,1 mm en época húmeda (octubre a mayo). De acuerdo con la clasificación ecológica propuesta por Holdridge, presenta las siguientes zonas de vida: bosque húmedo Montano Tropical (bh-MT), páramo muy húmedo Subalpino Tropical (pmh-SaT) y tundra pluvial Alpino Tropical (tp-AT) (ANA, 2010).

\section{Descripción de los sistemas de uso de suelo}

El bosque natural relicto de Japani es considerado el bosque natural relicto de queñuales más extenso de la sierra limeña, poblado principalmente por la especie Polylepis flavipila. Este bosque es aprovechado por los pobladores de la zona quienes extraen leña y plantas medicinales. Las áreas periféricas al bosque son utilizadas como zonas de pastoreo e incluso parte de estas áreas son destinadas para agricultura. El suelo es poco profundo, predominantemente litosólico (rocoso y pedregoso) con características superficiales paramosólicas, debido al gran espesor (colchón) compuesto básicamente por abundante materia orgánica en descomposición, proveniente fundamentalmente de la hojarasca (Curi, 2016).

El pastizal parcialmente compactado por sobrepastoreo abarca gran parte del área de investigación. La crianza del ganado es extensiva y se da a nivel familiar, siendo su objetivo la extracción de leche y obtención de carne, así como el de mantener las costumbres ancestrales, manifestadas en sus festividades. El tipo de ganado es vacuno en su totalidad, variando su número entre las cuatro mil a cinco mil cabezas. Entre los meses de abril a setiembre (época seca) el ganado se encuentra en la zona del bosque natural relicto de Japaní, ya sea en el área de bosque intervenido, en los suelos con pastizales parcialmente compactados o en los cultivos de papas en andenes agrícolas (post cosecha) mientras que los meses restantes (lluvias), sube a la parte más alta de la zona, colindante con los bofedales (Curi, 2016).

El área con cultivo de papas en andenes agrícolas, es la más pequeña de las 3 evaluadas. Se ubica en toda una ladera, entre los 3650-3800 msnm aproximadamente. Esta área, en conjunto con otras seis áreas cultivadas en otras laderas, se encuentra dispersa a lo largo y ancho del Bosque natural relicto de Japaní. El cultivo de papa se da bajo un sistema de agricultura de secano, con labranza manual y de rotación de cultivos en las diferentes laderas, permitiendo así descansar la tierra de dichas áreas alrededor de seis años cada una, facilitando el ciclo de recuperación del suelo gracias a la regeneración de la cobertura vegetal (principal fuente de fertilización de sus cultivos). En menor proporción, utilizan el estiércol seco del mismo ganado vacuno como abono. No obstante, esta práctica se ha ido perdiendo y la mayoría de comuneros solo deja descansar la tierra. Es importante añadir que, en los periodos de descanso de las 
áreas destinadas al cultivo de papa, son intervenidas por el ganado vacuno (Curi, 2016)

\section{Materiales y métodos}

El levantamiento de la información se llevó a cabo el mes de agosto (época seca) del año 2016. Se realizaron pruebas de infiltración de acuerdo a los tres usos de suelo, considerando además rangos altitudinales y pendientes fisiográficas. Siendo el factor suelo determinante en el estudio de la infiltración, se realizó una caracterización física química a partir de recolección de muestras y descripción de perfiles del suelo según sistema de uso.

Los materiales que se utilizaron en la investigación son los siguientes:

Pruebas de Infiltración: Cilindro infiltrómetro metálico de $12 \mathrm{~cm}$ de diámetro y $26 \mathrm{~cm}$ de alto, GPS, clinómetro, cronómetro, regla graduada, listón grueso de madera, comba, cuerdas, cilindros y baldes auxiliares, bolsas de plástico resistentes, libreta de campo y útiles de escritorio.

Caracterización físico química del suelo: Balanza digital, cilindro metálico pequeño, GPS, pala, pico, cinta métrica, bolsas ziploc de $1 \mathrm{~kg}$ de capacidad, bolsas de plástico resistentes, etiquetas, papel film, guantes, libreta de campo $\mathrm{y}$ útiles de escritorio.

\section{Pruebas de infiltración}

Se utilizó el método de cilindro infiltrómetro individual, aplicando la metodología indicada por Reyes (2014) debido a la practicidad del método, la portabilidad del equipo y la poca demanda de volumen de agua. El área de estudio es de difícil acceso (camino de herradura) con variaciones constante de pendientes fisiográficas a los largo del recorrido y con un solo punto de agua para el abastecimiento a los diferentes lugares de muestreo.

El estudio abarcó una pequeña área del Bosque natural relicto de Japaní procurando que esta, a pesar de su menor magnitud, mantenga la representatividad de cada uno de los 3 usos de suelo presentes. Posteriormente, se le aplicó el "muestreo aleatorio tipo bloques" generando en total 27 bloques distribuidos mediante tres criterios:

- Uso de suelo: tres grupos de tres bloques por cada uno de los tres usos de suelo

- Rango altitudinal: un grupo de tres bloques para la parte alta, uno en media y uno en baja, en cada uso de suelo.

- Pendiente fisiográfica: dentro de cada rango altitudinal de cada uso de suelo, un bloque con pendiente fisiográfica alta o mayor a $60^{\circ}$, otro con pendiente fisiográfica media entre $30^{\circ}$ y $60^{\circ}$ grados y el último con pendiente fisiográfica baja o menor a $30^{\circ}$.

Basado en el diseño muestral, se estableció un codificación para cada uso de suelo de acuerdo al rango altitudinal y a la pendiente fisiográfica que ocupo cada bloque. La descripción del código y la distribución de los bloques puede observarse en la Tabla 1 y Figura 2.
Tabla 1. Codificación de acuerdo a uso de suelo, altitud y pendiente

\begin{tabular}{cccc}
\hline Uso de suelo & Altitud & Pendiente & Código \\
\hline \multirow{3}{*}{ Bosque } & Alta & Alta & BA \\
& Medio & Media & BM \\
& Bajo & Baja & BB \\
\hline \multirow{3}{*}{ Pastizal } & Alta & Alta & PA \\
& Media & Media & PM \\
& Baja & Baja & PB \\
\hline \multirow{3}{*}{ Cultivo } & Alta & Alta & AA \\
& Media & Media & AM \\
& Baja & Baja & AB \\
\hline
\end{tabular}

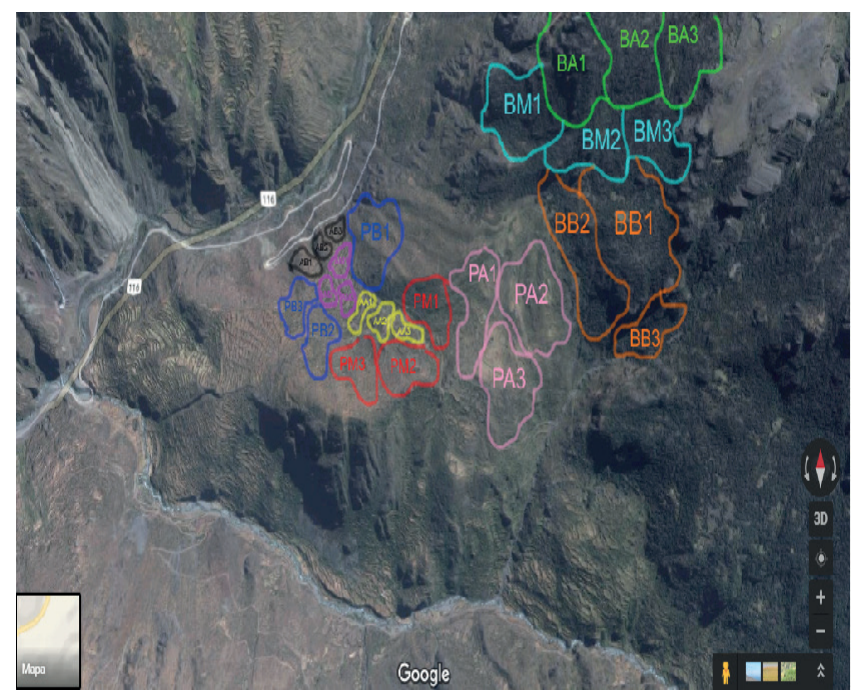

Figura 2. Distribución de bloques de acuerdo al uso de suelo, altitud y pendiente

De acuerdo al método utilizado, el cilindro infiltrómetro individual se introduce a una profundidad promedio de 10 $\mathrm{cm}$. Posteriormente se adhiere a la pared interna una regla graduada, para luego colocar una bolsa negra de plástico donde se vierte el agua. La prueba inicia a partir del retiro del plástico, siendo la primera lectura en regla en el tiempo cero. La duración de la prueba es de dos minutos, tomando lecturas cada cinco segundos. El objetivo de estos muestreos es determinar y comparar las capacidades de los distintos usos de suelo para poder absorber agua en un proceso de humedecimiento y sin intervención de la gravedad.

Touma et al. (2007) indica que, para cuantificar la cantidad de agua infiltrada en función al tiempo después de aplicar agua a la superficie del suelo, solo se necesitan dos características del suelo: la conductividad saturada del suelo y la sortividad. La primera es la tasa máxima de flujo de agua debido únicamente a la gravedad en un suelo completamente saturado y es una propiedad intrínseca del suelo. La segunda define la capacidad de un suelo para conducir agua por capilaridad, variando el contenido inicial y final de agua en el suelo.

Previo a la prueba de infiltración se determinó la humedad 
disponible del suelo por el método de bloques de yeso (Gonzales, 2015).

Con la información recogida, se elaboraron graficas de dispersión de datos para cada uno de los bloques. Cada grafica posee una ecuación de primer grado $(\mathrm{y}=\mathrm{bx}+\mathrm{a})$ cuya pendiente " $b$ " es considerada equivalente al valor de la "infiltración" y a su vez, sirve para diagnosticar si la infiltración fue buena o mala. El valor de la pendiente "b" corresponde a "lámina de agua infiltrada acumulada medida en centímetros" (eje Y de la Figura de dispersión) vs la "raíz del tiempo - medida en seg 0.5 " (eje X de la Figura de dispersión), por lo tanto "b" solo podrá obtener valores mayores a cero como mínimo o menores que la unidad como máximo (Figura 3).

Hasta la fecha no se cuenta con rangos de clasificación de esta, sin embargo, se tiene como referencia que valores entre 0,45 y $0,55\left(\mathrm{~cm} / \mathrm{seg}^{0.5}\right)$ son los óptimos para este parámetro (Alegre, 2013; citado por Reyes, 2014).

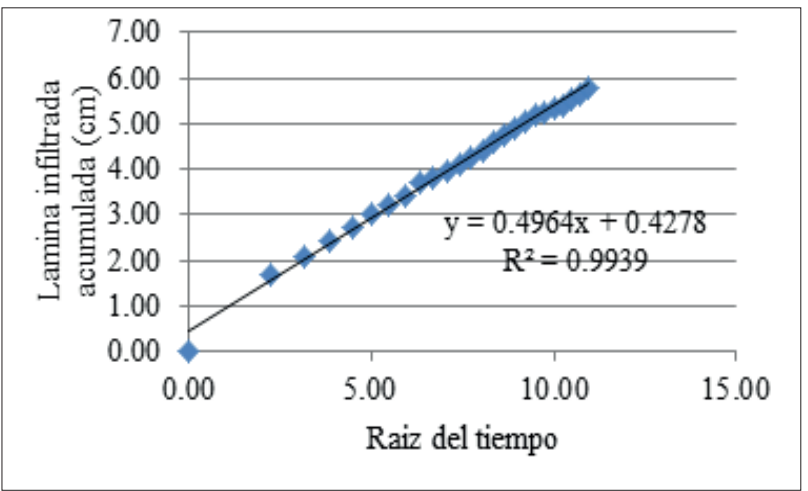

Figura 3. Infiltración $\left(\mathrm{cm} / \mathrm{seg}^{0.5}\right)$ en base a la ecuación de la recta

\section{Caracterización físico química del suelo}

Se hizo un croquis para cada una de las tres áreas de uso del suelo, con la finalidad de identificar sectores que presenten condiciones de paisaje (pendiente fisiográfica, exposición o drenaje) que puedan definir áreas con características distintivas (representativas). Se tomó un total de nueve muestras (tres por cada uso de suelo, teniendo en cuenta que una corresponda a la parte alta, otra a la parte media y la última a la parte más baja).

Los análisis fueron llevados a cabo en el laboratorio de análisis de suelos, plantas, agua y fertilizantes del departamento de suelos de la Facultad de Agronomía de la UNALM. Loso resultados se muestran en la Tabla 2 y Tabla 3 .

En el caso de la descripción de perfiles de suelo, se identificaron la estructura y profundidad de los diferentes horizontes de cada perfil, diferenciando las muestras por la presencia o ausencia de la capa de materia orgánica, la coloración in situ de los horizontes, presencia o ausencia de porosidad, grado de dureza, presencia o ausencia de proyecciones de raíces secundarias (Tabla 4). Por otro lado, en laboratorio se determinaron características edáficas, como la densidad aparente (Tabla 5) y el color del suelo (sistema de Munsell) (Tabla 6).

\section{Diseño estadístico}

Para poder interpretar los datos resultantes se utilizó el "Diseño Factorial 3k Proyectado". De acuerdo a este análisis, todos los factores en estudio están presentes en tres niveles. Es decir, la base o nivel siempre será tres y " $k$ " indica el número de factores. Particularmente en este estudio, coincide que los factores también son tres, siendo el primero el "uso de suelo" con los niveles "bosque intervenido", "pastizal parcialmente compactado por ganadería" y "cultivo de papas en andenes agrícolas"; mientras que el segundo factor es "la altitud" con los niveles "parte alta", "parte media" y "parte baja". Finalmente el último y tercer factor denominado "pendiente fisiográfica" presenta los niveles "pendiente fisiográfica alta", "pendiente fisiográfica media" y "pendiente fisiográfica baja".

Tabla 2. Resultados de las muestras de suelo según uso de selo vs textura

\begin{tabular}{|c|c|c|c|c|c|c|c|c|c|c|c|c|}
\hline \multirow[b]{2}{*}{ Uso de suelo } & \multirow[b]{2}{*}{ Cód } & \multirow[b]{2}{*}{$\begin{array}{l}\text { C.C } \\
(\% \mathrm{~V})\end{array}$} & \multirow[b]{2}{*}{$\begin{array}{c}\text { P.M } \\
(\% \mathrm{~V})\end{array}$} & \multicolumn{3}{|c|}{ Propiedades físicas según textura } & \multirow[b]{2}{*}{ C.I.C } & \multicolumn{5}{|c|}{ Cationes cambiables (meq/100g) } \\
\hline & & & & Adhesiv. & Infiltración & Aireación & & $\mathrm{Ca}^{2+}$ & $\mathrm{Mg}^{2+}$ & $\mathrm{K}^{+}$ & $\mathrm{Na}^{+}$ & $\frac{(\mathrm{AL})^{3+}}{(\mathrm{H})^{+}}+$ \\
\hline \multirow{4}{*}{ Bosque I. } & BA & 25,7 & 14,6 & Muy poco & Buena & Buena & 17,1 & 11,9 & 1,0 & 0,4 & 0,2 & 0,0 \\
\hline & BM & 28,9 & 16,6 & Muy poco & Buena & Buena & 21,8 & 13,9 & 1,0 & 0,5 & 0,1 & 0,0 \\
\hline & $\mathrm{BB}$ & 27,6 & 15,9 & Muy poco & Buena & Buena & 19,1 & 12,5 & 1,0 & 0,5 & 0,2 & 0,0 \\
\hline & PA & 35,0 & 20,4 & Media & Buena & Buena & 22,1 & 6,0 & 1,0 & 1,6 & 0,1 & 0,4 \\
\hline \multirow[t]{2}{*}{ Pastizal } & PM & 30,6 & 17,6 & Media & Buena & Buena & 20,3 & 11,8 & 1,8 & 2,7 & 0,1 & 0,0 \\
\hline & PB & 27,7 & 15,8 & Media & Buena & Buena & 17,3 & 9,9 & 1,5 & 1,7 & 0,1 & 0,0 \\
\hline \multirow{3}{*}{ Cultivo } & $\mathrm{AA}$ & 26,0 & 14,8 & Alta & Regular & Regular & 18,6 & 12,1 & 1,8 & 0,8 & 0,1 & 0,1 \\
\hline & $\mathrm{AM}$ & 27,1 & 15,5 & Alta & Regular & Regular & 18,2 & 13,7 & 1,7 & 1,5 & 0,1 & 0,0 \\
\hline & $\mathrm{AB}$ & 25,6 & 14,5 & Media & Buena & Buena & 18,1 & 12,2 & 2,1 & 2,3 & 0,1 & 0,0 \\
\hline
\end{tabular}


Tabla 3. Resultados de las muestras de suelo según uso de suelo vs fertilidad, análisis mecánico y textura

\begin{tabular}{|c|c|c|c|c|c|c|c|c|c|c|c|c|c|}
\hline \multirow{2}{*}{$\begin{array}{l}\text { Uso de } \\
\text { suelo }\end{array}$} & \multirow{2}{*}{ Código } & \multicolumn{6}{|c|}{ Fertilidad - características del suelo } & \multicolumn{3}{|c|}{ Análisis mecánico } & \multirow{2}{*}{ Textura } & \multirow{2}{*}{\multicolumn{2}{|c|}{ Clase textural }} \\
\hline & & $\mathrm{pH}$ & $\begin{array}{l}\text { C.E. } \\
(\mathrm{dS} / \mathrm{m})\end{array}$ & $\% \mathrm{M} . \mathrm{O}$ & $\mathrm{CaCO}_{3}$ & $\mathrm{P}(\mathrm{ppm})$ & $\mathrm{K}(\mathrm{ppm})$ & Arena & Limo & Arcilla & & & \\
\hline \multirow{3}{*}{ Bosque I. } & BA & 6,1 & 0,1 & 3,6 & 0,0 & 4,8 & 452.0 & 73.0 & 20.0 & 7.0 & Gruesa & $\begin{array}{c}\text { Arenoso } \\
\text { Franco }\end{array}$ & S. Arenoso \\
\hline & $\mathrm{BM}$ & 6,2 & 0,1 & 6,6 & 0,0 & 9,4 & 536.0 & 73.0 & 22.0 & 5.0 & Gruesa & $\begin{array}{c}\text { Arenoso } \\
\text { Franco }\end{array}$ & S. Arenoso \\
\hline & $\mathrm{BB}$ & 5,5 & 0,1 & 7,8 & 0,0 & 13,0 & 1302.0 & 72.0 & 22.0 & 6.0 & Gruesa & $\begin{array}{l}\text { Arenoso } \\
\text { Franco }\end{array}$ & S. Arenoso \\
\hline \multirow{3}{*}{ Pastizal } & PA & 5,4 & 0,1 & 7,5 & 0,0 & 33,7 & 224.0 & 63.0 & 30.0 & 7.0 & $\begin{array}{l}\text { Moderadamente } \\
\text { Gruesa }\end{array}$ & $\begin{array}{l}\text { Franco } \\
\text { Arenoso }\end{array}$ & S. Franco \\
\hline & PM & 6,3 & 0,3 & 6,5 & 0,0 & 58,4 & 738.0 & 59.0 & 28.0 & 13.0 & $\begin{array}{l}\text { Moderadamente } \\
\text { Gruesa }\end{array}$ & $\begin{array}{l}\text { Franco } \\
\text { Arenoso }\end{array}$ & S. Franco \\
\hline & PB & 6,1 & 0,2 & 4,6 & 0,0 & 29,6 & 359.0 & 53.0 & 28.0 & 19.0 & $\begin{array}{c}\text { Moderadamente } \\
\text { Gruesa }\end{array}$ & $\begin{array}{l}\text { Franco } \\
\text { Arenoso }\end{array}$ & S. Franco \\
\hline \multirow{3}{*}{ Cultivo } & $\mathrm{AA}$ & 5.4 & 0,2 & 3,6 & 0,0 & 9,0 & 132.0 & 53.0 & 24.0 & 23.0 & $\begin{array}{l}\text { Moderadamente } \\
\text { Fina }\end{array}$ & $\begin{array}{l}\text { Franco } \\
\text { Arcilloso } \\
\text { Arenoso }\end{array}$ & S. Franco \\
\hline & $\mathrm{AM}$ & 6,1 & 0,4 & 4,1 & 0,0 & 23,6 & 125.0 & 53.0 & 26.0 & 21.0 & $\begin{array}{l}\text { Moderadamente } \\
\text { Fina }\end{array}$ & $\begin{array}{l}\text { Franco } \\
\text { Arcilloso } \\
\text { Arenoso }\end{array}$ & S. Franco \\
\hline & $\mathrm{AB}$ & 6,0 & 0,3 & 4,8 & 0,0 & 34,8 & 119.0 & 59.0 & 24.0 & 17.0 & $\begin{array}{l}\text { Moderadamente } \\
\text { Gruesa }\end{array}$ & $\begin{array}{c}\text { Franco } \\
\text { Arenoso }\end{array}$ & S. Franco \\
\hline
\end{tabular}

Tabla 4. Características visuales dentro de los perfiles de suelo

\begin{tabular}{ccc}
\hline Uso de suelo & Estrato 1 & Estrato 2 \\
\hline Bosque & $\begin{array}{c}\text { Capa de materia orgánica de } 7 \mathrm{~cm} \text { de profundidad. Textura } \\
\text { media }\end{array}$ & $\begin{array}{c}6 \mathrm{~cm} \text { de profundidad. Baja proporción de pedregosidad e } \\
\text { intermedia presencia de raicillas. Textura media }\end{array}$ \\
Pastizal & $\begin{array}{c}4 \mathrm{~cm} \text { de profundidad. Ausencia de capa orgánica marcada } \\
\text { sobre el suelo. Presencia intermedia de raicillas }\end{array}$ & $\begin{array}{c}16 \mathrm{~cm} \text { de profundidad. Alta presencia de raicillas. } \\
\text { Presencia de poros. Textura muy fina }\end{array}$ \\
Cultivo & $\begin{array}{c}3 \mathrm{~cm} \text { de profundidad. Ausencia evidente de capa orgánica } \\
\text { sobre el suelo. Constituida por fragmentos aglomerados de } \\
\text { suelo. Textura muy gruesa }\end{array}$ & $\begin{array}{c}24 \mathrm{~cm} \text { de profundidad. Presencia de raicillas del cultivo } \\
\text { (papa). Alta proporción de poros. Textura gruesa }\end{array}$ \\
\hline Uso de suelo & Estrato 3 & Estrato 4 \\
\hline Bosque & $\begin{array}{c}13 \mathrm{~cm} \text { de profundidad. Pedregosidad intermedia y alta } \\
\text { presencia de raicillas. Presencia de raíces de 1 cm de } \\
\text { diámetro. Textura fina }\end{array}$ & $\begin{array}{c}\text { Desde los 28 cm de profundidad en adelante. Alta } \\
\text { proporción de pedregosidad y baja presencia de raicillas. } \\
\text { Presencia de raíces de 2 cm de diámetro. Textura fina }\end{array}$ \\
Pastizal & $\begin{array}{c}\text { Desde los 21 cm de profundidad en adelante. Presencia } \\
\text { baja de raicillas. Presencia de manchas blanquecinas }\end{array}$ & \\
Cultivo & Desde los 28 cm en adelante. Alta pedregosidad. & \\
\hline
\end{tabular}

Tabla 5. Densidad aparente según los perfiles de suelo

\begin{tabular}{cccc}
\hline Uso de suelo & Estrato & D.A g/ $\mathrm{cm}^{3}$ & D.A g/cm ${ }^{3}$. Prom \\
\hline \multirow{3}{*}{ Bosque } & 1 & 0,30 & \\
& 2 & 0,67 & 0,63 \\
& 3 & 0,79 & \\
Pastizal & 4 & 0,76 & 0.62 \\
& 1 & 0,28 & \\
& 2 & 0,76 & 0.78 \\
Cultivo & 3 & 0,83 & \\
& 1 & 0,68 & \\
\hline
\end{tabular}


Tabla 6. Color del suelo (Munsell)

\begin{tabular}{|c|c|c|c|}
\hline Capas/uso de suelo & Color (Munsell) & Nombre del color & Tonalidad \\
\hline Estrato 1 - Bosque intervenido & $10 \mathrm{YR} 3 / 2$ & Pardo grisáceo muy oscuro & \\
\hline Estrato 2 - Bosque intervenido & $10 \mathrm{YR} 3 / 2$ & Pardo grisáceo muy oscuro & \\
\hline Estrato 3 - Bosque intervenido & $10 \mathrm{YR} 4 / 2$ & Pardo grisáceo oscuro & \\
\hline Estrato 4 - Bosque intervenido & $10 \mathrm{YR} 4 / 2$ & Pardo grisáceo oscuro & \\
\hline Estrato 1 - Pastizal parcialmente compactado por ganaderia & 10 YR 4/2 & Pardo grisáceo oscuro & \\
\hline Estrato 2 - Pastizal parcialmente compactado por ganaderia & $10 \mathrm{YR} 4 / 2$ & Pardo grisáceo oscuro & \\
\hline Estrato 3 - Pastizal parcialmente compactado por ganaderia & 10 YR 5/2 & Pardo grisáceo & \\
\hline Estrato 1 - Cultivo de papas en andenes agricolas & $10 \mathrm{YR} 5 / 2$ & Pardo grisáceo & \\
\hline Estrato 2 - Cultivo de papas en andenes agricolas & $10 \mathrm{YR} 5 / 2$ & Pardo grisáceo & \\
\hline Estrato 3 - Cultivo de papas en andenes agricolas & $10 \mathrm{YR} 5 / 2$ & Pardo grisáceo & \\
\hline
\end{tabular}

\section{Resultados y discusión}

Los resultados de las pruebas de infiltración se detallan en la Tabla 7.

Tabla 7. Resultados de infiltración $\left(\mathrm{cm} / \mathrm{seg}^{0.5}\right)$ según el uso de suelo, rango altitudinal y pendiente fisiográfica

\begin{tabular}{ccccc}
\hline \multicolumn{2}{c}{ Uso del suelo } & Bosque & Pastizal & Cultivo \\
\hline \multirow{4}{*}{ Altitud alta } & Pendiente alta & 0.66 & 0.43 & 0.31 \\
& Pendiente media & 0.25 & 0.40 & 0.23 \\
& Pendiente baja & 0.17 & 0.22 & 0.17 \\
& Pendiente alta & 0.39 & 0.50 & 0.23 \\
Altitud media & Pendiente media & 0.22 & 0.39 & 0.22 \\
& Pendiente baja & 0.13 & 0.26 & 0.40 \\
& Pendiente alta & 0.48 & 0.38 & 0.22 \\
& Pendiente media & 0.35 & 0.37 & 0.38 \\
& Pendiente baja & 0.29 & 0.37 & 0.36 \\
\hline
\end{tabular}

Tabla 8. Variables estadísticas obtenidas a partir de los datos de infiltración

\begin{tabular}{cccc}
\hline Uso de Suelo & Bosque & Pastizal & Cultivo \\
\hline Número de datos & 9 & 9 & 9 \\
Media & 0.33 & 0.37 & 0.28 \\
Varianza & 0.0276 & 0.0071 & 0.0070 \\
$\begin{array}{c}\text { Desviación Estándar } \\
\text { Límite máximo de la } \\
\text { desviación }\end{array}$ & 0.1662 & 0.0840 & 0.0837 \\
$\begin{array}{c}\text { Límite mínimo de la } \\
\text { desviación }\end{array}$ & 0.16 & 0.49 & 0.36 \\
\hline
\end{tabular}

En la Tabla 7 se evidencia que, el mayor valor promedio de infiltración, según los usos de suelo, se dio en el pastizal parcialmente compactado por sobrepastoreo con un valor de $0.37 \mathrm{~cm} / \mathrm{seg}^{0.5}$, seguido del bosque intervenido con $0.33 \mathrm{~cm} / \mathrm{seg}^{0.5}$ y finalmente el cultivo de papas en andenes agrícolas que registró $0.28 \mathrm{~cm} / \mathrm{seg}^{0.5}$. Es importante, indicar que de acuerdo a la desviación estándar (Tabla 8) los valores obtenidos para el uso de suelo bosque intervenido presenta mayor dispersión frente a su valor promedio, lo que también puede observarse en la representación gráfica obtenida a partir de las muestras (Figura 4).

Hewlett (1982) afirma que, en suelos compactados y cultivados, se presentan niveles bajos de infiltración, mientras que esto no ocurre en suelos forestales. No obstante, cuando el suelo forestal es alterado por actividades agropecuarias se presentan problemas en la infiltración, ya que el efecto directo generado por su tala se refleja en la pérdida de cobertura y por ende de la capacidad de retención de agua en el suelo.

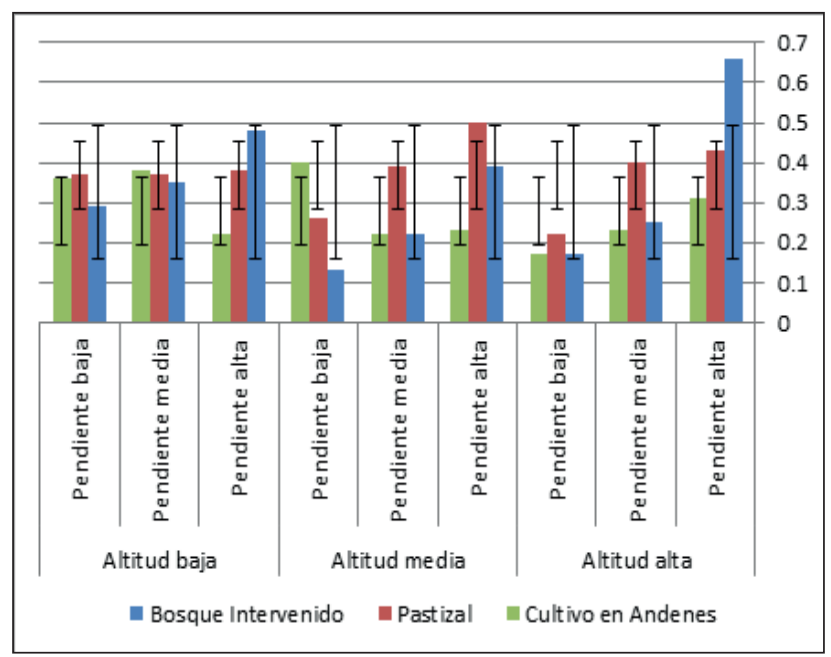

Figura 4. Representación gráfica de los resultados de infiltración, destacando la desviación estandar y sus respectivos límites de confianza.

Tomando como referencia el rango de valores óptimos establecido por Alegre en el 2013 0,45-0,55 cm/seg ${ }^{0.5}$, únicamente los resultados provenientes de los puntos: "parte alta del bosque intervenido con pendiente fisiográfica alta"; "'parte media del pastizal parcialmente compactado por sobrepastoreo con pendiente fisiográfica alta" y "parte 
baja del bosque intervenido con pendiente fisiográfica alta" serían considerados como poseedores de una óptima infiltración, siendo $0,66 \mathrm{~cm} / \mathrm{seg}^{0.5}, 0,50 \mathrm{~cm} / \mathrm{seg}^{0.5} \mathrm{y} 0,48 \mathrm{~cm} /$ seg $^{0.5}$ los respectivos valores de esta.

$\mathrm{Si}$ bien los 3 resultados con valores óptimos de infiltración proveniente de dos diferentes usos de suelo (bosque intervenido y pastizal parcialmente compactado porsobrepastoreo) y de dos rangos altitudinales distintos (parte alta y media), todos comparten el mismo rango de pendiente fisiográfica, la cual es de tipo alta $\left(>60^{\circ}\right)$.

No se presentó ningún valor óptimo de infiltración en ningún punto muestreado del uso de suelo "Cultivo de papas en andenes agrícolas".

Reyes (2014) utilizó el método del cilindro infiltrómetro individual para determinar la infiltración en diferentes sistemas de uso del suelo ubicados en el IRD Fundo la Génova (Chanchamayo, Junín, Selva Central), obteniendo una infiltración promedio de $0,42 \mathrm{~cm} / \mathrm{seg}^{0.5}$ para la plantación de Tectona grandis de 28 años de edad con altitud aproximada de 850 m.s.n.m. y $27^{\circ}$ de pendiente fisiográfica ; $0,49 \mathrm{~cm} /$ $\operatorname{seg}^{0.5}$ para los cítricos con altitud aproximada de $(850$ m.s.n.m) y $8^{\circ}$ de pendiente fisiográfica $; 0,39 \mathrm{~cm} / \mathrm{seg}^{0.5}$ para la plantación agroforestal con 6 años de abandono (Coffea arábica e Inga edulis), altitud aproximada de 900 m.s.n.m. y $6^{\circ}$ de pendiente fisiográfica. Si bien es dificil poder realizar una comparación con estos resultados, debido a las diferencias considerables en cuánto a características propias de las zonas y sobretodo por la falta de semejanza entre los sistemas de uso de suelo comparados, es uno de los pocos registros en el que se relaciona y compara la infiltración de diferentes sistemas de uso del suelo, utilizando la misma metodología.

Blas y Mejia (2014) declaran que, la materia orgánica en el suelo es la responsable de brindar energía a los microorganismos, los cuales son los primeros responsables de la agregación del mismo. A su vez, si se presenta mayor cantidad de materia orgánica en determinado suelo, posteriormente se generará una mayor producción de ácidos fúlvicos y húmicos, los cuales al igual que los microorganismos, estabilizan la estructura del suelo. Es así que a los microorganismos y a los mayores contenidos de materia orgánica se les considera "inhibidores de la destrucción de los agregados". Estos agregados al no destruirse, mantienen una adecuada estructura que implica una mayor capacidad de infiltración. Finalmente Díaz (2007) citado por Blas y Mejia (2014) añade que, la materia orgánica favorece la producción de porosidad y en consecuencia, la capacidad de infiltración. En síntesis, a mayor cantidad de materia orgánica, mayor cantidad de infiltración en el suelo, ya sea por ser fuente de alimento para los microorganismos en constante movimiento, por la producción de ácidos fúlvicos-húmicos o por generar vacíos al descomponerse.

Los resultados de los análisis de suelos (Tabla 3) son coherentes con la teoría expuesta ya que el uso "pastizal parcialmente compactado por sobrepastoreo" (infiltración de $0,37 \mathrm{~cm} / \mathrm{seg}^{0.5}$ ) evidenció un promedio de materia orgánica de 6,2\%, mientras que el "bosque intervenido" (infiltración de $0,33 \mathrm{~cm} / \mathrm{seg}^{0.5}$ ) presentó $6,0 \% \mathrm{y}$, finalmente el uso de suelo "cultivo de papas en andenes agrícolas" (infiltración de $0,28 \mathrm{~cm} / \mathrm{seg}^{0.5}$ ), obtuvo en promedio $4,2 \%$ de materia orgánica.

En el caso del pastizal, el valor obtenido de materia orgánica, pudo deberse a dos razones: las características propias de las pasturas de la zona, cuyos ciclos vegetativos cortos permiten la acumulación de considerables cantidades de materia orgánica en los primeros centímetros del suelo, proveniente de la abundante cantidad de raicillas que estos presentan. En segunda instancia, a la mayor ocurrencia y cantidad de excrementos de ganado distribuidos a lo largo de todo el área, los cuales son una de las fuentes principales de materia orgánica en suelos intervenidos.

Si promediamos los 27 resultados de infiltración, agrupándolos únicamente por "tipo de pendiente fisiográfica" se obtienen valores de infiltración de $0,40 \mathrm{~cm} /$ seg $^{0.5}$ para los puntos evaluados con pendiente fisiográfica tipo "Alta"; $0,31 \mathrm{~cm} / \mathrm{seg}^{0.5}$ para el promedio de puntos con pendiente fisiográfica tipo "Media" y finalmente $0,26 \mathrm{~cm} /$ $\mathrm{seg}^{0.5}$ para el promedio de puntos que se encuentran dentro de la clasificación pendiente fisiográfica "Baja".

Si bien ninguno de los 3 promedios se encuentra dentro de la clasificación de valores óptimos de infiltración propuesta por Alegre $\left(0,45-0,55 \mathrm{~cm} / \mathrm{seg}^{0.5}\right)$, sí se encuentra una relación (de tipo directamente proporcional) entre la pendiente fisiográfica y la infiltración. En otras palabras, a mayor pendiente fisiográfica se presentó mayor infiltración.

Adicionalmente, si promediamos los 27 resultados, asociándolos por "tipo de pendiente fisiográfica dentro de cada diferente uso de suelo", se obtienen los valores representados en la Tabla 9.

Tabla 9. Promedio de infiltración según pendiente fisiográfica por cada uso de suelo

\begin{tabular}{cccc}
\hline $\begin{array}{c}\text { Promedio de Infiltración } \\
\left(\mathrm{cm} / \mathrm{seg}^{0.5}\right) \\
\text { (Pendiente fisiográfica// intervenido } \\
\text { Uso) }\end{array}$ & $\begin{array}{c}\text { Bosque } \\
\text { parcialmente } \\
\text { compactado } \\
\text { por } \\
\text { sobrepastoreo }\end{array}$ & $\begin{array}{c}\text { Cultivo de } \\
\text { papas en } \\
\text { andenes } \\
\text { agrícolas }\end{array}$ \\
\hline Pendiente Alta & 0,44 & 0,51 & 0,25 \\
Pendiente Media & 0,38 & 0,27 & 0,28 \\
Pendiente Baja & 0,28 & 0,20 & 0,31 \\
\hline
\end{tabular}

Para los usos de suelo "bosque intervenido" y "pastizal parcialmente compactado por sobrepastoreo", los valores promedio de infiltración incrementan a medida que la pendiente se hace más pronunciada. Esta relación directa no se manifiesta en el cultivo de papas en andenes agrícolas muy probablemente porque la diferenciación entre rangos de pendiente para dicho cultivo no tuvo la significancia que sí se dio en los otros usos de suelo, debido a su propia naturalidad y condiciones óptimas de siembra, la cual no requiere de pendientes pronunciadas.

Finalmente, al agrupar los resultados de infiltración según similitud de "rango altitudinal" dentro de cada tipo de 
"pendiente fisiográfica", se obtienen los resultados de la Tabla 10.

Tabla 10. Infiltración según rango altitudinal por cada tipo de pendiente fisiográfica

\begin{tabular}{|c|c|c|c|}
\hline $\begin{array}{l}\text { Promedio de infiltración } \\
\left(\mathrm{cm} / \mathrm{seg}^{0.5}\right) \\
\text { (Altitud//Pendiente }\end{array}$ & $\begin{array}{l}\text { Pendiente } \\
\text { fisiográfica }\end{array}$ & $\begin{array}{l}\text { Pendiente } \\
\text { fisiográfica } \\
\text { Media }\end{array}$ & $\begin{array}{l}\text { Pendiente } \\
\text { fisiográfica } \\
\text { Baia }\end{array}$ \\
\hline fisiográfica) & & & \\
\hline Parte Alta & 0.47 & 0.29 & 0.19 \\
\hline Parte Media & 0.36 & 0.36 & 0.34 \\
\hline Parte Baja & 0.37 & 0.28 & 0.26 \\
\hline
\end{tabular}

La Tabla 10 evidencia que los resultados de infiltración poseen una relación directa al asociar el rango altitudinal y tipo de pendiente fisiográfica. Es decir, a medida que la pendiente fisiográfica es mayor (dentro de cada rango altitudinal) el valor de la infiltración también se hace mayor.

Esta relación entre "rango altitudinal" y el "tipo de pendiente fisiográfica", es la única (entre todas las combinaciones posibles de los tres parámetros evaluados) en la que, el parámetro "altitud" permite generar una relación (en este caso, directamente proporcional) con los resultados de la infiltración, gracias a que actúa en compañía e influencia de la "pendiente fisiográfica". Sin embargo, no se encontró relación alguna a mayor o menor altitud dentro de cada rango de pendiente.

En cuanto a los resultados obtenidos del análisis de suelos (Tabla 2 y 3 ) se demuestra que en seis de las nueve zonas evaluadas (exceptuando la parte baja del bosque intervenido y las partes altas del pastizal parcialmente compactado por ganadería y cultivo de papas en andenes agrícolas) se presentan las mejores condiciones para la asimilación de nutrientes y desarrollo de las plantas (por poseer un $\mathrm{pH}$ entre 6,0 y 7,5). Además, por lo expuesto por Bruns \& Martin (1986) citado por Gonzales (2014), en las mismas seis zonas se dan condiciones deseables de abundancia y presencia de lombrices de tierra dentro del suelo, ya que esta condición sobresale en un rango de $\mathrm{pH}$ de 6,0-7,0

En general, los pastizales fueron los que presentaron los mayores contenidos de materia orgánica con un promedio de $6,18 \%$ entre sus tres rangos altitudinales, seguidos por el uso de suelo bosque intervenido con $5,99 \%$ y finalmente la zona de cultivo de papas en andenes agrícolas con 4,20\%.

La determinación del color del suelo se realizó empleando las cartillas de color del suelo Munsell con muestra seca, cuyos resultados se muestran en la Tabla 6. En general, los colores variaron del pardo grisáceo muy oscuro al pardo grisáceo. Para Craul (s.f.) es más común encontrar las coloraciones oscuras o parduzcas en las zonas superficiales del suelo. Además agrega que esto puede ser causado por un mayor contenido de materia orgánica muy descompuesta, cuyo color se intensifica al incrementar la humificación. Finaliza al declarar que, el efecto que tiene la materia orgánica sobre la coloración de los suelos es más marcado en los arenosos.
De acuerdo a la Tabla 5, y obteniendo el valor promedio de densidad aparente para cada tipo de uso de suelo, se registran los siguientes valores para bosque intervenido $0,63 \mathrm{~g} / \mathrm{cm}^{3}$, mientras que el promedio del uso de suelo pastizal parcialmente compactado por ganadería fue de $0,62 \mathrm{~g} / \mathrm{cm}^{3}$ y finalmente, el uso de suelo cultivo de papas en andenes agrícolas reporto un valor de $0,78 \mathrm{~g} / \mathrm{cm}^{3}$.

Rubio (2010) agrega que los suelos bien estructurados y con altos contenidos de materia orgánica presentan valores más bajos de densidad aparente que los suelos poco estructurados y con bajos contenidos de materia orgánica. Esto es consecuente con lo ocurrido ya que el uso de suelo pastizal parcialmente compactado por ganadería obtuvo el mayor contenido de materia orgánica, con un promedio de $6,18 \%$ y una densidad aparente promedio de $0,62 \mathrm{~g} / \mathrm{cm}^{3}$ mientras que el uso de suelo bosque intervenido presentó $5,90 \%$ de contenido de materia orgánica y $0,63 \mathrm{~g} / \mathrm{cm}^{3}$ de densidad aparente; finalmente el uso de suelo cultivo de papas en andenes agrícolas con 4,2\% de materia orgánica y $0,78 \mathrm{~g} / \mathrm{cm}^{3}$ de densidad aparente.

\section{Conclusiones}

Los usos de suelo "bosque intervenido" y "pastizal parcialmente compactado por sobrepastoreo" fueron los únicos con valores óptimos de infiltración, siendo estos; $0,66 \mathrm{~cm} / \mathrm{seg}^{0.5}$ y $0,48 \mathrm{~cm} / \mathrm{seg}^{0.5}$ para los puntos muestreados dentro de la parte "alta" y "baja" del "bosque intervenido con pendiente fisiográfica alta"; así como $0,50 \mathrm{~cm} /$ $\mathrm{seg}^{0.5}$ para la parte "media" del "pastizal parcialmente compactado por ganadería con pendiente fisiográfica alta". El uso de suelo "pastizal parcialmente compactado por sobrepastoreo" posee mayor cantidad de infiltración promedio $0,7 \mathrm{~cm} / \mathrm{seg}^{0.5}$ en contraste con el "bosque intervenido" $0,33 \mathrm{~cm} / \mathrm{seg}^{0.5}$ a causa de su menor humedad inicial disponible en el suelo al momento de las pruebas $30,6 \%$ frente a $49,4 \%$ del bosque.

De acuerdo a los resultados estadísticos, la pendiente fisiográfica, especialmente sobre los $60^{\circ}$, resultó ser el factor que más influye en la infiltración, lo cual se refleja en los promedios de infiltración por tipo de pendiente fisiográfica: $0,47 \mathrm{~cm} / \mathrm{seg}^{0.5}$ para pendiente alta; $0,33 \mathrm{~cm} /$ $\mathrm{seg}^{0.5}$ para pendiente intermedia y $0,27 \mathrm{~cm} / \mathrm{seg}^{0.5}$ para pendiente baja. A su vez, la "altitud" solo mostró un efecto significativo sobre los resultados de infiltración al actuar en conjunto con el factor "pendiente fisiográfica".

Ademas, los mayores valores de infiltración en altas pendientes causados por su mayor capacidad de enraizamiento; los altos contenidos de materia orgánica de acelerada descomposición causante de una mayor frecuencia de espacios porosos, sus raíces profundas, la abundante cantidad de macrofauna y el mayor contenido de hojarasca en su suelo forestal dado por sus copas densas, ratificaron que, las características propias del uso de suelo bosque en Japaní permiten las mejores condiciones para el proceso de infiltración. Por otro lado, el uso de suelo cultivo de papas en andenes agrícolas manifiesta una 
pérdida considerable de estas propiedades.

Finalmente, se hace pertinente asegurar que, confiando en la correcta toma de datos de humedad inicial en campo $\mathrm{y}$ apelando a lo enunciado por Llerena et al (2017), el uso de suelo con "bosque intervenido", dentro del área de Japaní, desempeña un papel importante en la protección de la cabecera de cuenca, de los arroyos y laderas de la zona, caracterizándose por almacenar agua en el subsuelo, incluyendo la época seca, permitiendo su distribución a lo largo del año gracias a la paulatina infiltración, percolación y demás procesos subsiguientes que las características intrínsecas del mismo permiten.

\section{Literatura citada}

Aquafondo. 2016. Inversión En Agua Para Lima. Recuperado el 15 de enero de 2017. Disponible en: http://aquafondo.org.pe/.

ANA - Autoridad Nacional del Agua. 2010. Evaluación de los Recursos Hídricos de la Cuenca del Río Rímac: Estudio Hidrológico y Ubicación de la Red de Estaciones Hidrométricas en la Cuenca del Río Rímac. Recuperado el 5 de diciembre de 2016. Disponible en: http:// www.ana.gob.pe/sites/default/files/publication/files/1 estudio_hidrologico_cuenca_rimac_-_volumen_i_-_ texto_-_final_2010_0.pdf.

Blas, E. \& Mejía, AR. 2014. Cobertura vegetal y capacidad de infiltración de agua en el suelo (Tesis de pregrado) UNASAM, Ancash.

Craul, PJ. (s/f). Urban soils (en línea). Estados Unidos. North Carolina State University. P. 45-61. Recuperado 10 noviembre 2016. Disponible en: https:/www.ces. ncsu.edu/fletcher/programs/nursery/metria/metria05/ m57.pdf.

Curi, M. 2016. Entrevista personal del 03 de enero del 2016.

Doornbos, E. 2015. El valor de los bosques andinos en asegurar agua y suelo en un contexto de creciente riesgo climático:¿(re)conocemos lo imperdible?. Disponible en:http://www.bosquesandinos.org/wp-content/ uploads/2015/12/061115_articulo_n3.pdf.

FAO - Food and Agriculture Organization for the United Nations. Afrontar la escasez del agua. Un marco de acción para la agricultura y la seguridad alimentaria. Recuperado el 01 de noviembre de 2017. Disponible en: http://www.fao.org/3/a-i3015s.pdf.

Gonzalez, GJ. 2015. Caracterización de la infiltración en bosques plantados con Polylepis spp., de 11 y 29 años, Parque Nacional Huascarán, quebrada Quilcayhuanca, Huaraz, Áncash. (Tesis de pregrado). UNALM, Lima.

Hewlett, JD. 1982. Principles of Forest Hydrology. Recuperado el 30 de junio de 2016, de https://www. usask.ca/watershed/pdf/Aberdeen_evening_reading/ Hewlett.pdf.

INEI - Instituto Nacional de Estadística e Informática. Precipitación total anual, según departamento, 20012015. Recuperado el 20 de enero de 2017. Disponible en: tematico/climate/.

https://www.inei.gob.pe/estadisticas/indice-

Llerena, CA., Hermoza, RM., Yalle, SR., Flores, F y Salinas, N. 2017. Forest Management and Water in Peru. UNESCO 1(37): 128-148.

Reyes, RJ. 2014. Influencia de cuatro sistemas del uso de tierras (teca, cítricos y café bajo sombra manejado y en abandono), en la sortividad en el IRD Selva Fundo la Génova, Chanchamayo, Junín (Tesis de pregrado). UNALM, Lima.

Rubio Gutierrez, AM. 2010. La densidad aparente en suelos forestales del parque natural Los Arconocales (en línea). España. Universidad de Sevilla. 88 p. Recurperado el 20 noviembre 2016. Disponible en: http://digital. csic.es/bitstream/10261/57951/1/La\%20densidad $\% 20$ aparente $\% 20$ en $\% 20$ suelos\%20forestales $\% 20$.pdf.

Sembrero, MP y Valencia, N. 2015. Expediente técnico Área de conservación privada "Japaní", Carampoma, Huarochirí. Lima, PE. 45 p.

Touma, J., Voltz, M y Alberger, J. 2007. Determining soil saturated hydraulic conductivity and sorptivity from single ring infiltration tests. Wiley Online Library (58): 229-238.

Vásquez, A. 2011. Sistema de captación del agua de lluvia en laderas semiáridas de la sierra y su impacto en el proceso de desertificación y cambio climático (Tesis doctoral). UNALM, Lima. 